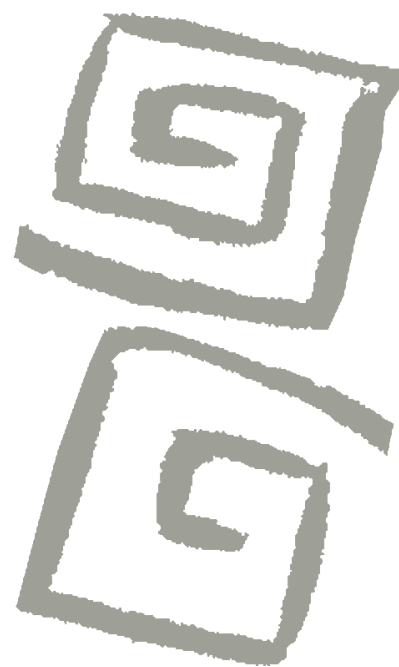

\title{
Concentración y desigualdades en el financiamiento de las obras sociales posdesregulación: un análisis comparativo de los años 2004 y 2011
}

\author{
Concentration and inequalities in the financing \\ of obras sociales after deregulation: a \\ comparative analysis of the years 2004 and 2011
}

María Florencia Arnaudo ${ }^{1}$, Fernando Lago ${ }^{2}$, Nebel Moscoso ${ }^{3}$, Ernesto Báscolo ${ }^{4}$, Natalia Yavich ${ }^{5}$

'Licenciada en Economía, Magister en Ingeniería de Procesos Petroquímicos. Becaria, Consejo Nacional de Investigaciones Científicas y Técnicas (CONICET). Asistente de Docencia, Instituto de Investigaciones Económicas y Sociales del Sur, Universidad Nacional del Sur, Argentina. marnaudo@uns.edu.ar

²icenciado en Economía, Doctor en Economía. Investigador, Consejo Nacional de Investigaciones Científicas y Técnicas (CONICET).

Profesor Adjunto, Instituto de Investigaciones Económicas y Sociales del Sur, Universidad Nacional del Sur, Argentina.

flago@uns.edu.ar

${ }^{3}$ Licenciada en Economía, Doctora en Economía. Investigadora Adjunta, Consejo Nacional de Investigaciones Científicas y Técnicas (CONICET). Profesora Asociada Universidad Nacional del Sur, Argentina.nmoscoso1@gmail. com

${ }^{4}$ Licenciado en Economía, Doctor en Ciencias Sociales. Investigador Adjunto, Consejo Nacional de Investigaciones Científicas y Técnicas (CONICET), Argentina. ebascolo@gmail.com

${ }^{5}$ Licenciada en Ciencias Antropológicas, Doctora en Ciencias Sociales. Investigadora Asistente, Consejo Nacional de Investigaciones Científicas y Técnicas (CONICET), Argentina. nyavich@gmail.com
RESUMEN En Argentina, durante la década de 1990 se introdujeron grandes cambios en el marco regulatorio de las obras sociales nacionales. A partir de datos de la Administración Federal de Ingresos Públicos, el presente estudio compara para los años 2004 y 2011: a) el peso de las obras sociales dentro del sistema de salud argentino; b) el grado de concentración de este subsistema de la seguridad social en salud; y c) la desigualdad en la disponibilidad de fondos per cápita entre obras sociales y beneficiarios. Los resultados revelan un incremento de la importancia de las obras sociales dentro del sistema de salud; la cantidad de instituciones se mantuvo prácticamente invariante y la concentración de los fondos distribuidos en las entidades más importantes del subsistema no muestra cambios, mientras que la de cotizantes aumenta levemente y disminuye la de beneficiarios. Además, se registró una reducción en las desigualdades en los fondos por beneficiario, la cual podría estar asociada a la atenuación de las diferencias salariales entre los diferentes sectores de la economía y al accionar del Fondo Solidario de Redistribución.

PALABRAS CLAVES Instituciones de Seguridad Social; Asignación de Recursos para la Atención de Salud; Regulación Gubernamental; Argentina.

\begin{abstract}
In Argentina, during the decade of the 1990s major changes were introduced into the regulatory framework of the national obras sociales, or union-based health coverage plans. Using data from the Federal Administration of Public Income (AFIP) [Administración Federal de Ingresos Públicos], this study evaluates for the years 2004 and 2011: a) the importance of obras sociales within the healthcare system, b) the degree of concentration of this health social security subsystem, and c) the inequalities in the availability of funds among the obras sociales and their beneficiaries. The results show an increased importance of obras sociales within the Argentine health system. The concentration of funds distributed to the most important institutions within the subsystem showed no change, while the concentration of contributors to these institutions slightly increased and that of beneficiaries decreased. Finally, a reduction of the inequalities in funds per beneficiary received by different institutions was observed. This trend can be explained, among other factors, by the attenuation of wage differentials between branches of economic activity and the actions of the so-called Solidarity Redistribution Fund.
\end{abstract}

KEY WORDS Social Security Agencies; Health Care Rationing; Government Regulation; Argentina. 


\section{INTRODUCCIÓN}

El sistema de salud argentino está compuesto por tres subsistemas: el público, destinado principalmente a la población de menores ingresos sin cobertura de salud; el de la seguridad social, que brinda cobertura a asalariados integrados a la economía formal y su grupo familiar; y el privado, que atrae mayormente a la población de medianos y altos ingresos y provee aseguramiento voluntario a cambio del pago de una prima ${ }^{(1),(2)}$.

El subsistema de la seguridad social se consolidó como tal con la sanción de la Ley 18610 en el año 1970, que estableció un seguro social compulsivo para todos los asalariados y su grupo familiar, el cual sería financiado con un aporte personal y una contribución patronal calculados como un porcentaje fijo del salario(3).

Este subsistema se organizó en torno a instituciones denominadas obras sociales, organizaciones sin fines de lucro, oferentes de seguros de salud. En el caso de las denominadas obras sociales nacionales, su gerenciamiento quedó en manos de los sindicatos representativos de cada rama de actividad de la economía. Adicionalmente, existen obras sociales provinciales, que brindan cobertura a los empleados públicos de cada jurisdicción, obras sociales del personal de dirección, y el Instituto Nacional de Seguridad Social para Jubilados y Pensionados (INSSJP), que brinda cobertura de los servicios de salud en forma exclusiva a jubilados y pensionados.

La Ley 18610 también instituyó un organismo regulador, el Instituto Nacional de Obras Sociales, encargado de controlar las prestaciones y el financiamiento, y de establecer mecanismos de solidaridad entre las distintas aseguradoras del sistema. Una herramienta clave para alcanzar este último objetivo fue la creación del denominado Fondo Solidario de Redistribución, al cual todas las entidades estaban obligadas a contribuir con un $10 \%$ de sus ingresos. Este fondo estaba destinado a disminuir las diferencias de recursos existentes entre las obras sociales, derivadas -entre otros factores- de los diferentes salarios promedio de cada rama de actividad. Con este fin debía apoyar financieramente a las obras sociales de menores ingresos, de forma tal de garantizar a sus afiliados las prestaciones de servicios de salud y el financiamiento de planes especiales ${ }^{(4)}$.

Hasta mediados de la década de 1980 la evolución del subsistema de seguridad social exhibió una constante ampliación de la cobertura, favorecida por un contexto económico en el que la mayoría de la fuerza laboral se insertaba en el mercado formal: mientras que en 1967 solamente el 35\% de la población argentina poseía obra social, tal cobertura alcanzó en el año 1984 el $75 \%{ }^{(5)}$. Sin embargo, hacia fines de la misma década se cuestionó fuertemente la eficiencia de las entidades y la presencia de desigualdades en el financiamiento y la cobertura de servicios de salud entre beneficiarios de distintas obras sociales $^{(6)}$

Los problemas de eficiencia se explicaban por diferentes causas. La ausencia de competencia entre entidades impedía que los beneficiarios del sistema pudiesen elegir la obra social de su preferencia, dado que eran afiliados en forma compulsiva a una entidad, según su rama de actividad. A esto se sumaba la debilidad regulatoria del Instituto Nacional de Obras Sociales, que se mostró incapaz de limitar la cobertura de servicios innecesarios ofrecidos por las entidades más ricas, y de garantizar una cobertura mínima a los beneficiarios de las entidades más pobres ${ }^{(5)}$. Se esgrimía que estos motivos generaban un bajo nivel de eficiencia, que era responsable de las deudas adquiridas con los prestadores de servicios ${ }^{(7)}$ y del continuo crecimiento de la planta de personal de las entidades, que se consideraba excesiva con relación a la cantidad de afiliados ${ }^{(5),(8)}$.

Las desigualdades en el financiamiento también se atribuían a la obligatoriedad de afiliación por rama de actividad, lo cual segmentaba a la población asalariada en función de su capacidad contributiva, con fuertes contrastes en la cobertura ofrecida entre distintas obras sociales ${ }^{(6)}$. A su vez, la utilización de mecanismos discrecionales en la asignación del Fondo Solidario de Redistribución limitaba su efectividad redistributiva. 
En este contexto, entre los años 1993 y 1999 se aplicaron una serie de reformas al sector de la seguridad social, que alcanzaron a la gran mayoría de las obras sociales nacionales. Solo quedaron excluidas del proceso de desregulación las obras sociales nacionales de las fuerzas de seguridad y de las universidades. Por su parte, las obras sociales provinciales quedaron al margen, al estar sujetas a las normas de cada gobierno subnacional. Los objetivos planteados, y las medidas específicas encaminadas a su concreción se resumen a continuación:

\section{Aumentar la eficiencia del subsistema} mediante la introducción de mecanismos de competencia entre obras sociales ${ }^{(2)}$. Se desregula el sistema de obras sociales nacionales y se dota de libertad de elección a sus afiliados, quienes podrían cambiar de aseguradora una vez al año. Sin embargo, y luego de diversos cambios, finalmente se estableció que la elección quedaba limitada a las obras sociales del mismo tipo, no siendo posible el traspaso de una obra social nacional sindical a otra de personal de dirección o viceversa, ni desde una obra social a una empresa de medicina prepaga.

2. Propiciar niveles más igualitarios de cobertura mediante la definición de un paquete mínimo de prestaciones, conocido como Programa Médico Obligatorio. Debía ser garantizado por todas las entidades aseguradoras $y$, en el caso de que alguna no pudiera cumplirlo, tendría que fusionarse con otra/s entidades.

3. Atenuar las desigualdades en los ingresos por afiliado entre las obras sociales, garantizando un piso en los aportes de los cotizantes del sistema. De esta manera, todas las entidades podrían cubrir el costo de la canasta básica de prestaciones incluidas en el Programa Médico Obligatorio. Con este fin, el Decreto 292/95 redefine los mecanismos de reparto del Fondo Solidario de Redistribución, asegurando a todo beneficiario titular del sistema una cotización mínima. Cuando los aportes de un trabajador en función de su salario fuesen menores a dicho valor mínimo, el Fondo Solidario de Redistribución aportaría en forma automática la diferencia. Además, una parte de este fondo financiaría tratamientos costosos de baja frecuencia incorporados en la Administración de Programas Especiales $^{(3)}$.

Es importante mencionar que, aunque no hubo una incorporación explícita de los seguros privados en la desregulación, la firma de convenios prestacionales por los cuales algunas obras sociales nacionales transfieren los aportes de sus afiliados a una empresa de medicina prepaga (la cual se responsabiliza por la provisión de los servicios de salud y/o el gerenciamiento de la cartera de beneficiarios) permitió que estas ingresaran al sistema de la seguridad social, compitiendo por la captación de afiliados con el resto de las entidades ${ }^{(9)}$. La posibilidad de pactar planes diferenciales de salud entre los beneficiarios y las obras sociales sirvió de medio para que esto ocurriera ${ }^{(10)}$. Sin embargo, este tipo de afiliados (denominados por las empresas de medicina prepaga "asociados indirectos") conservan su carácter de cotizantes de la seguridad social.

Se esperaba que la introducción de la competencia promoviese la eficiencia del sistema. Con el nuevo régimen, los afiliados que no estuvieran satisfechos con la calidad y cobertura de las prestaciones ofrecidas por su entidad podrían ejercer el derecho al traspaso. Si una obra social experimentaba una pérdida de afiliados significativa, eventualmente debía cesar sus actividades. De esta manera, al incentivar la concentración de los usuarios en un número menor de entidades, se pretendía alcanzar mayores economías de escala, una reducción de los costos administrativos y una mayor diversificación de los riesgos dentro de cada institución.

Un aspecto a destacar de los cambios regulatorios era el conflicto potencial entre la introducción de competencia entre entidades (derivada de la libertad de elección) y los objetivos de aumento de cobertura y la atenuación de las desigualdades en el financiamiento. 
Findling et al. ${ }^{(6)}$ analizaron los efectos tempranos de la desregulación. Utilizando datos del año 1999 hallaron que, si bien el número de usuarios que ejercieron la opción de traspaso fue reducido en relación al total, la proporción de trabajadores que cambiaron de una obra social nacional a otra aumentaba a medida que lo hacía el nivel salarial. Asimismo, Fidalgo ${ }^{(9)}$ provee datos que indican que, en el periodo comprendido entre el año 1999 y el 2005, ocho de las diez obras sociales nacionales que lideraban el ranking de traspasos recibidos poseían convenios con empresas de medicina prepaga. Por último, Cetrángolo y Devoto ${ }^{(10)}$ afirman que la eliminación en el año 1997 de las obras sociales del personal de dirección del régimen de desregulación, se debió a que estas estaban captando la mayor parte de los traspasos. En conjunto, estos resultados sugieren que en los primeros años de la desregulación existió un descreme del mercado en beneficio de las entidades más "ricas" en términos relativos o que poseían convenios con empresas de medicina prepaga. De persistir esta tendencia, podría suponer un desfinanciamiento progresivo de las obras sociales más pobres, a partir de la pérdida de sus afiliados de mayores salarios ${ }^{(6)}$.

En la década posterior a la sanción de las reformas, la combinación de una serie de factores podría haber limitado las tendencias pro-desigualdad en el financiamiento, derivadas de la libertad de elección entre obras sociales.

En primer lugar, la dinámica del mercado de trabajo en este periodo se caracterizó por la notable reducción de la desigualdad salarial, la cual obedece en mayor medida a la más intensa recomposición de ingresos en la parte inferior de la distribución ${ }^{(11)}$. Esta dinámica estuvo marcada por la combinación de un contexto económico altamente favorable con un fuerte proceso de crecimiento y niveles de desocupación en descenso, así como por los cambios regulatorios del mercado laboral introducidos a partir de la sanción de la Ley 25877, que modificó sensiblemente el marco de las convenciones colectivas de trabajo, fortaleciendo la capacidad de negociación de los sindicatos.
En segundo lugar, los criterios de reparto del Fondo Solidario de Redistribución y su estructura de financiamiento fueron progresivamente transformados: en el año 2002, se incrementaron los porcentajes de aportes, que se diferencian de acuerdo al salario de los cotizantes; luego, mediante el Decreto 741/2003 se pasó de garantizar una cotización mínima por cada trabajador titular, a una situación en la que se garantizaba un piso por beneficiario (que incluía al trabajador titular y sus dependientes). De esta forma, los grupos familiares más numerosos tendrían asegurado un piso de aportes mayor. Posteriormente, en 2006, el Decreto 1901 crea el Subsidio Automático Nominativo que introduce un ajuste por riesgo en la cotización mínima asegurada por cada integrante del grupo familiar (incluido el titular) conforme a su sexo y edad, con el objetivo de evitar la selección por riesgos por parte de las aseguradoras. Las cotizaciones mínimas fijadas en el decreto original fueron actualizadas posteriormente en los años 2010 y $2011^{(3),(10)}$ (Cuadro 1).

El presente estudio se plantea como objetivos comparar para los años 2004 y 2011: a) el peso de las obras sociales nacionales dentro del sistema de salud argentino; b) el grado de concentración de este subsistema de la seguridad social en salud, en relación con los fondos recibidos y la cantidad de instituciones, beneficiarios y cotizantes; y c) la desigualdad en la disponibilidad de fondos per cápita entre obras sociales nacionales y beneficiarios.

\section{METODOLOGÍA}

Se llevó a cabo un estudio observacional descriptivo y retrospectivo basado en un análisis de datos cuantitativos relacionado con las obras sociales nacionales, correspondientes a los años 2004 y 2011. La selección de los datos del período en cuestión se debe a que si bien la implementación efectiva de la opción de cambio se dio con el Decreto 504/98, el período comprendido entre los años 1999 y 2002 estuvo signado por la crisis 
Cuadro 1. Cotizaciones mínimas (en pesos) del Fondo Solidario de Redistribución. Argentina, años 2006, 2010 y 2011.

\begin{tabular}{lcccccc}
\hline \multirow{2}{*}{$\begin{array}{l}\text { Grupo de } \\
\text { edad }\end{array}$} & \multicolumn{2}{c}{$\mathbf{2 0 0 6}$} & \multicolumn{2}{c}{$\mathbf{2 0 1 0}$} & \multicolumn{2}{c}{$\mathbf{2 0 1 1}$} \\
\cline { 2 - 6 } & $\begin{array}{c}\text { Hombres } \\
\text { (\$ pesos) }\end{array}$ & $\begin{array}{c}\text { Mujeres } \\
(\$ \text { pesos) }\end{array}$ & $\begin{array}{c}\text { Hombres } \\
\text { (\$ pesos) }\end{array}$ & $\begin{array}{c}\text { Mujeres } \\
\text { (\$ pesos) }\end{array}$ & $\begin{array}{c}\text { Hombres } \\
\text { (\$ pesos) }\end{array}$ & $\begin{array}{c}\text { Mujeres } \\
\text { (\$ pesos) }\end{array}$ \\
\hline $\mathbf{0 - 1 4}$ & 22,0 & 22,0 & 36,0 & 36,0 & 47,0 & 47,0 \\
$\mathbf{1 5 - 4 9}$ & 35,0 & 41,0 & 57,0 & 67,0 & 74,0 & 87,0 \\
$\mathbf{5 0 - 6 4}$ & 41,0 & 41,0 & 67,0 & 67,0 & 87,0 & 87,0 \\
Más de 65 & 99,5 & 99,5 & 148,0 & 148,0 & 192,0 & 192,0 \\
\hline
\end{tabular}

Fuente: Programa de las Naciones Unidas para el Desarrollo ${ }^{(3)}$.

económica más profunda de la historia argentina, lo cual limitó el funcionamiento de la seguridad social a su mínimo nivel. Recién a partir del año 2003 es posible considerar que las reformas implementadas tienen impacto sobre la movilidad de beneficiarios y aportes entre las obras sociales.

A lo largo del trabajo, se entiende por afiliado o beneficiario de las obras sociales nacionales a la suma de los cotizantes y los adicionales, siendo los cotizantes los trabajadores empleados en el sistema formal (y de cuyo salario se descuenta el aporte respectivo a la obra social). Mientras que los adicionales son aquellas personas que reciben la cobertura de la obra social por ser familiar directo (hijo, cónyuge, madre o nieto a cargo) del cotizante, pero no realizan un aporte con salarios propios a la obra social del cotizante.

Sin bien hubiese sido deseable discriminar en el estudio el porcentaje de los cotizantes de obras sociales nacionales que trasladan su aporte a una empresa de medicina prepaga, tal proceder no resulta factible. Aunque el censo 2010 desglosa la población que accede a una empresa de medicina prepaga a través de una obra social nacional, no ocurre lo mismo con los datos de la Administración Federal de Ingresos Públicos (AFIP), principal fuente de información utilizada.

En cuanto al primer objetivo, para dimensionar la participación de las obras sociales nacionales se analizó el peso relativo (en términos de porcentaje del gasto total en salud que representa y población cubierta) de: a) el subsector de la seguridad social sobre el conjunto del sistema de salud, y b) las obras sociales nacionales desreguladas en relación al conjunto de entidades del subsector de la seguridad social.

La información del gasto total en salud por subsector se tomó del Global Health Observatory Data Repository de la Organización Mundial de la Salud (OMS) ${ }^{(12)}$. Los datos de cantidad de habitantes se obtuvieron del Censo Nacional de Población, Hogares y Viviendas de 2001 y de 2010 y los datos de población cubierta por subsistema y tipo de obra social se obtuvieron, para el 2010, del informe del Programa de Naciones Unidas para el Desarrollo ${ }^{(3)} y$, para 2005, de Cavagnero et al. ${ }^{(15)}$. Con respecto a los datos de cobertura, ante la imposibilidad de obtener información para el año 2004, se tomaron los datos del año 2005.

El segundo objetivo plantea estudiar la evolución del grado de concentración del subsistema de obras sociales nacionales, dado que este era el mecanismo a partir del cual se esperaba aumentar la eficiencia promedio de las entidades. Con este fin, se midió en cada año analizado: i) la cantidad de obras sociales nacionales sujetas a desregulación, conjuntamente con el número de beneficiarios y cotizantes, y ii) los porcentajes de beneficiarios, cotizantes y fondos distribuidos acaparados por las cinco, diez y veinte entidades desreguladas más importantes, ordenadas según el número de cotizantes. Estos datos fueron extraídos del Informe de seguridad social: subsistemas de la seguridad social de la AFIP(16). 
Dado que la información es provista en forma mensual, para contar con datos anualizados se procedió a obtener el acumulado anual sumando los fondos distribuidos a las obras sociales nacionales en los 12 meses del año. Para calcular la cantidad de cotizantes y beneficiarios de cada obra social nacional se consideró en cada año el promedio simple de los datos declarados en forma mensual. En cada año analizado, se excluyeron del estudio aquellas entidades que no registraron continuidad en sus actividades durante los doce meses ${ }^{[a]}$.

En cuanto al tercer objetivo, las desigualdades en el financiamiento de las obras sociales nacionales fueron estudiadas utilizando los siguientes datos: a) los fondos anuales distribuidos, desagregados por entidad, que incluyen las transferencias netas del Fondo Solidario de Redistribución (fondos girados por el Fondo Solidario de Redistribución a las obras sociales menos los aportes de las obras sociales al Fondo); b) los promedios anuales de cotizantes y beneficiarios por obras sociales nacionales; c) el salario promedio anual por cotizante por entidad (estimado a partir del promedio simple de los datos mensuales para cada año analizado).

Los datos obtenidos fueron analizados mediante técnicas descriptivas de concentración y distribución. Para analizar las desigualdades en la disponibilidad de fondos entre las obras sociales nacionales, se las agrupó en quintiles según el salario promedio por cotizante. Para cada quintil de entidades se determinó: a) el porcentaje que representan los beneficiarios de las obras sociales de cada quintil sobre el total del sistema, b) el promedio de fondos distribuido por cotizante y por beneficiario, c) el número promedio de beneficiarios por cotizante y d) el promedio simple del salario medio del cotizante para cada grupo de entidades.

Una debilidad del análisis anterior es que pone en pie de igualdad a todas las obras sociales, independientemente de su cantidad de afiliados. De esta forma, en el cálculo de los promedios tiene el mismo peso una entidad con 300.000 afiliados que otra con 5.000. Con el objeto de superar esta falencia, se analizan las desigualdades en la disponibilidad de fondos entre el conjunto de los afiliados al sistema, agrupándolos en quintiles según el valor del salario promedio por beneficiario, determinados por su obra social de pertenencia. Para cada quintil de beneficiarios, se calculó el porcentaje del total de fondos distribuidos que le corresponde. Adicionalmente, se computó el coeficiente de Gini como medida sintética del grado de desigualdad en la distribución de los fondos.

\section{RESULTADOS}

\section{Relevancia de las obras sociales nacionales en el sistema de salud argentino}

Considerando que el financiamiento público del gasto total en salud comprende tanto al subsector de la seguridad social como al público, en el Cuadro 2 puede observarse un aumento del $16 \%$ entre los años 2004 y 2011. Esto ocurre a expensas de una reducción de igual magnitud en el porcentaje de participación en el gasto total en salud del gasto privado (que incluye gasto de bolsillo y gasto en seguros privados). La dinámica del financiamiento público se explica, en mayor medida, por la expansión del financiamiento del subsector de la seguridad social (que incluye a las obras sociales nacionales, el Instituto Nacional de Seguridad Social para Jubilados y Pensionados y las obras sociales provinciales) que aumenta un $14 \%$.

Cuadro 2. Distribución porcentual del gasto en salud, según subsector. Argentina, 2004 y 2011.

\begin{tabular}{lcc}
\hline Subsector & $\begin{array}{c}\mathbf{2 0 0 4} \\
\mathbf{( \% )}\end{array}$ & $\begin{array}{c}\mathbf{2 0 1 1} \\
\mathbf{( \% )}\end{array}$ \\
\hline Seguridad social & 29,0 & 43,0 \\
Sector público & 22,0 & 24,0 \\
Privado & 49,0 & 33,0 \\
\end{tabular}

Fuente: Elaboración propia a partir de datos del Global Health Observatory Data Repository ${ }^{(12)}$. 
Cuadro 3. Distribución porcentual de la población, según cobertura de salud. Argentina, 2005 y 2010 .

\begin{tabular}{lcc}
\hline & 2005 & 2010 \\
Tipo de cobertura & $\mathrm{n}=38.592 .150$ & $\mathrm{n}=40.117 .096$ \\
& $(\%)$ & $(\%)$
\end{tabular}

$\begin{array}{lrr}\text { Seguridad social } & & \\ \text { Obra social nacional } & 32,0 & 39,0 \\ \text { INSSJyP } & 8,0 & 8,0 \\ \text { Obra social provincial } & 14,0 & 14,0 \\ \text { Sector público } & 39,0 & 30,0 \\ \text { Seguros voluntarios } & 7,0 & 9,0\end{array}$

Fuente: Elaboración propia a partir de los totales poblacionales del Instituto Nacional de Estadística y Censos ${ }^{(13),(14)}$, de datos del PNUD ${ }^{(3)}$ para 2005 y de Cavagnero et al. ${ }^{(15)}$ para 2010.

INSSJyP = Instituto Nacional de Seguridad Social para Jubilados y Pensionados. asalariado registrado $^{[\mathrm{b}]}$ (considerando tanto el empleo público como el privado) aumentó del $60 \%$ en mayo del 2002 al $64 \%$ en el segundo trimestre de $2012^{(17)}$.

Para determinar la importancia de las obras sociales nacionales sujetas a desregulación dentro del subsector de la seguridad social, se calculó qué porcentaje representan sus beneficiaros con relación a la población total de Argentina (Cuadro 3).

Se observa un aumento en la importancia de este subconjunto de instituciones en términos del porcentaje de la población cubierta, teniendo como contrapartida la reducción de la población sin ningún tipo de cobertura explícita, atendida por la red de centros de salud pertenecientes al sector público. Asimismo, la proporción de la población que posee seguros voluntarios en el sector privado también registra un incremento cercano al $2 \%$.

\section{Desregulación y concentración}

En el Cuadro 4 se exhibe la cantidad de obras sociales nacionales y el promedio anual de cotizantes y beneficiarios en cada uno de los años analizados, así como el cambio porcentual de cada variable en el período bajo análisis.

Contrariamente a lo esperado, el número de obras sociales nacionales (con continuidad de aportes a lo largo de cada año) aumenta

Cuadro 4. Número de obras sociales nacionales, promedio anual de cotizantes y beneficiarios, y variación porcentual entre 2004 y 2011. Argentina.

\begin{tabular}{lrrr}
\hline & $\mathbf{2 0 0 4}$ & $\mathbf{2 0 1 1}$ & $\begin{array}{c}\text { Variación } \\
\mathbf{( \% )}\end{array}$ \\
\hline Obras sociales nacionales & 288 & 293 & 1,7 \\
Cotizantes & 3.974 .593 & 6.530 .894 & 64,3 \\
Beneficiarios & 7.331 .138 & 9.666 .417 & 31,8 \\
& & & \\
\hline
\end{tabular}

Fuente: Elaboración propia a partir de datos de la Administración Federal de Ingresos Públicos ${ }^{(16)}$. 
Cuadro 5. Porcentaje de cotizantes, beneficiarios y fondos distribuidos en obras sociales nacionales con mayor número de beneficiarios. Argentina, 2004 y 2011.

\begin{tabular}{|c|c|c|c|c|c|c|}
\hline \multirow{2}{*}{$\begin{array}{l}\text { OSN posicionadas } \\
\text { según número de } \\
\text { beneficiarios }\end{array}$} & \multicolumn{2}{|c|}{$\begin{array}{c}\text { Cotizantes } \\
(\%)\end{array}$} & \multicolumn{2}{|c|}{$\begin{array}{c}\text { Beneficiarios } \\
(\%)\end{array}$} & \multicolumn{2}{|c|}{$\begin{array}{c}\text { Fondos distribuidos } \\
\text { (\%) }\end{array}$} \\
\hline & 2004 & 2011 & 2004 & 2011 & 2004 & 2011 \\
\hline 5 primeras & 29,0 & 35,0 & 35,0 & 33,0 & 29,0 & 30,0 \\
\hline 10 primeras & 44,0 & 47,0 & 47,0 & 45,0 & 44,0 & 45,0 \\
\hline 20 primeras & 59,0 & 62,0 & 62,0 & 59,0 & 62,0 & 60,0 \\
\hline
\end{tabular}

Fuente: Elaboración propia a partir de datos de la Administración Federal de Ingresos Públicos ${ }^{(16)}$. OSN= Obras sociales nacionales.

levemente. Asimismo, la cantidad de cotizantes del sistema exhibe un incremento del $64,3 \%$, acompañado por un aumento (aunque de menor magnitud) en la cantidad de beneficiarios.

La mayor cantidad de cotizantes es consistente con la dinámica del mercado laboral en el periodo analizado, con una importante caída en los niveles de desempleo y el crecimiento de la población económicamente activa y la formalidad laboral ${ }^{(18)}$. El menor incremento en el número de beneficiarios con respecto al de cotizantes puede explicarse por la existencia de más de un cotizante por grupo familiar (que evita la necesidad de incluir a todo el grupo familiar en una única obra social) y/o la incorporación al mercado de trabajo de jóvenes sin hijos.

En el Cuadro 5 se analizan las tendencias a la concentración en las principales entidades del sistema (según número de beneficiarios) en lo que respecta a cotizantes, beneficiarios y fondos distribuidos.

No existe una tendencia claramente definida en lo relativo al grado de concentración del sistema. Mientras que la de los cotizantes aumenta en todas las categorías consideradas, la de beneficiarios cae levemente (entre el $2 \%$ y el $3 \%$ ). Asimismo, el porcentaje de los fondos percibidos por las entidades más importantes según el número de beneficiarios prácticamente no muestra cambios.

\section{Distribución de los fondos de las obras sociales nacionales}

El Cuadro 6 agrupa las obras sociales en quintiles según el salario promedio de los cotizantes de cada entidad para los años 2004 y 2011. Se observa que el porcentaje de afiliados adherido a las obras sociales del primer y segundo quintil (las más pobres en términos relativos) se reduce en un $9 \%$ y un $7 \%$ respectivamente. Al mismo tiempo los tres quintiles superiores aumentan su cobertura en un $2 \%$, un $8 \%$ y un $4 \%$, respectivamente.

La diferencia entre los fondos por beneficiarios de las obras sociales del primer y quinto quintil se reduce de 2,94 veces en 2004 a 2,44 en 2011 (17\%). Las desigualdades en los fondos por cotizantes entre los mismos quintiles siguen una evolución similar, cayendo un 9,5\%.

Se observa también una disminución de las diferencias en el salario medio del cotizante entre quintiles extremos (28\%), lo cual puede explicar (al menos parcialmente) la evolución en las desigualdades de los fondos por beneficiario y por cotizante. Sin embargo, y en la medida en que para cada año analizado las diferencias salariales superan a las registradas en los fondos por cotizante, se hace evidente el rol desempeñado por el Fondo Solidario de Redistribución en la atenuación de las desigualdades en el financiamiento. 
Cuadro 6. Distribución porcentual del total de beneficiarios, de los fondos por beneficiario, y de los fondos y adicionales por cotizante, según quintiles de obras sociales ordenadas por salario medio del cotizante. Argentina, 2004 y 2011.

\begin{tabular}{|c|c|c|c|c|c|c|}
\hline Año & $\begin{array}{c}\text { Quintiles de } \\
\text { obras sociales }\end{array}$ & $\begin{array}{c}\text { Total de } \\
\text { beneficiarios } \\
(\%)\end{array}$ & $\begin{array}{c}\text { Fondos por } \\
\text { beneficiario }^{1} \\
(\$ \text { pesos })\end{array}$ & $\begin{array}{c}\text { Fondos por } \\
\text { cotizante }^{2} \\
(\$ \text { pesos })\end{array}$ & $\begin{array}{l}\text { Adicionales } \\
\text { por cotizante } \\
(\$ \text { pesos })\end{array}$ & $\begin{array}{l}\text { Salario medio } \\
\text { del cotizante } \\
\quad(\$ \text { pesos })\end{array}$ \\
\hline \multirow[t]{5}{*}{2004} & 1 & 43,3 & 395,68 & 672,11 & 0,70 & 552,56 \\
\hline & 2 & 17,6 & 442,79 & 851,22 & 0,92 & 870,65 \\
\hline & 3 & 13,0 & 540,56 & $1.091,60$ & 1,02 & $1.127,94$ \\
\hline & 4 & 16,6 & 804,42 & $1.582,51$ & 0,97 & $1.620,57$ \\
\hline & 5 & 9,5 & $1.162,67$ & $2.354,75$ & 1,03 & $3.482,68$ \\
\hline \multirow[t]{5}{*}{2011} & 1 & 34,5 & $2.080,05$ & $2.744,83$ & 0,32 & $2.273,38$ \\
\hline & 2 & 11,3 & $2.448,59$ & $3.578,02$ & 0,46 & $3.474,02$ \\
\hline & 3 & 15,3 & $2.716,38$ & $4.214,65$ & 0,55 & $4.213,37$ \\
\hline & 4 & 25,0 & $3.425,68$ & $5.454,26$ & 0,59 & $5.779,96$ \\
\hline & 5 & 13,9 & $5.085,34$ & $8.696,65$ & 0,71 & $10.299,61$ \\
\hline
\end{tabular}

Fuente: Elaboración propia a partir de datos de la Administración Federal de Ingresos Públicos ${ }^{(16)}$.

${ }^{1}$ Cociente quintil 5 y quintil $1=2,94$ en 2004 y 2,44 en 2011.

${ }^{2}$ Cociente quintil 5 y quintil $1=3,50$ en 2004 y 3,17 en 2011.

${ }^{3}$ Cociente quintil 5 y quintil $1=6,30$ en 2004 y 4,53 en 2011.

Los adicionales por cotizantes muestran una reducción en todos los quintiles en el periodo analizado. Esto puede ser una consecuencia de los cambios demográficos intercensales (2001-2010) en los que la población menor de 14 años se redujo del $28,3 \%$ al $25,5 \%$, mientras que la población entre 15 y 64 años se incrementó del $61,8 \%$ al $64,3 \%$. Tal comportamiento demográfico tiene su correlato en la paridez media final ${ }^{[c]}$, que para el total del país ha descendido desde 3,1 hijos por mujer en el 2001 a 2,9 en 2010, lo cual evidencia un descenso de la fecundidad en la última década.

El Cuadro 7 compara la evolución de los fondos distribuidos entre los beneficiarios del sistema. Los beneficiarios de las obras sociales nacionales son agrupados en quintiles según el valor de los fondos promedio por beneficiario, el cual está determinado por su obra social de pertenencia. De esta forma,
Cuadro 7. Porcentajes del total de los fondos distribuidos según quintil de beneficiarios. Argentina, 2004 y 2011.

\begin{tabular}{ccc}
\hline $\begin{array}{c}\text { Quintiles de } \\
\text { beneficiarios }\end{array}$ & \multicolumn{2}{c}{$\begin{array}{c}\text { Total de fondos } \\
\text { distribuidos }\end{array}$} \\
\cline { 2 - 3 } & $\begin{array}{c}\mathbf{2 0 0 4} \\
\mathbf{( \% )}\end{array}$ & $\begin{array}{c}\mathbf{2 0 1 1} \\
\mathbf{( \% )}\end{array}$ \\
\hline 1 & 11,0 & 13,0 \\
2 & 14,0 & 15,0 \\
3 & 15,0 & 18,0 \\
4 & 22,0 & 22,0 \\
5 & 38,0 & 32,0 \\
\hline & & \\
\hline $\begin{array}{l}\text { Fuente: Elaboración propia a partir de datos de la Administración } \\
\text { Federal de Ingresos Públicos }{ }^{(16)} \text {. }\end{array}$ \\
${ }^{1}$ Cociente quintil 5 y quintil 1 3,48 en 2004 y 2,54 en 2011.
\end{tabular}


el primer quintil está compuesto por el 20\% de los beneficiarios que reciben la menor cantidad de fondos en términos per cápita, mientras que el quinto quintil está constituido por el $20 \%$ con mayores fondos per cápita.

Con base en los datos analizados, es posible afirmar que entre los años 2004 y 2011 las diferencias en los fondos recibidos por los beneficiarios del primer y quinto quintil tienden a reducirse. Sin embargo, tal reducción se explica en gran medida por la caída del $6 \%$ en la participación en los fondos recibidos por los afiliados del quinto quintil. En efecto, los beneficiarios del primero, si bien ven aumentado el porcentaje de fondos que reciben, solo registran un incremento del $2 \%$.

Una mejor medida de la evolución de las desigualdades en la distribución de fondos entre beneficiarios de las obras sociales nacionales puede obtenerse a partir de la confección de curvas de Lorenz para cada año analizado, y el cálculo de los correspondientes coeficientes de Gini, lo cual permite obtener un panorama global que considera todos los quintiles de beneficiarios, en lugar de considerar únicamente los extremos. El coeficiente de Gini de la distribución de fondos entre los beneficiarios de las obras sociales nacionales, para el año 2004, fue de 0,246 mientras que, para el año 2011, fue de 0,185 valores que, junto al análisis de las curvas de Lorenz (Figura 1), confirman la tendencia positiva en lo relativo a la atenuación de las desigualdades.

\section{CONCLUSIONES Y DISCUSIÓN}

El análisis desarrollado revela un aumento de la importancia relativa del subsector de la seguridad social dentro del sistema de salud argentino en el periodo analizado. Tal conclusión se sustenta en el incremento del porcentaje del gasto total en salud que financia este subsector, así como por la expansión de la población a la que da cobertura. En particular, mientras que el porcentaje de la población incluida en el Instituto Nacional de Seguridad Social para Jubilados y Pensionados registra un incremento marginal, y tanto la población asegurada por las obras sociales provinciales como la atendida en forma exclusiva por el subsector público se reducen levemente en términos relativos, la proporción de la población cubierta por las obras sociales nacionales (sujetas a desregulación) se incrementa en un 7\% entre los años 2005 y 2010. Sin embargo, una debilidad de este análisis surge al omitir (por ausencia de información) la

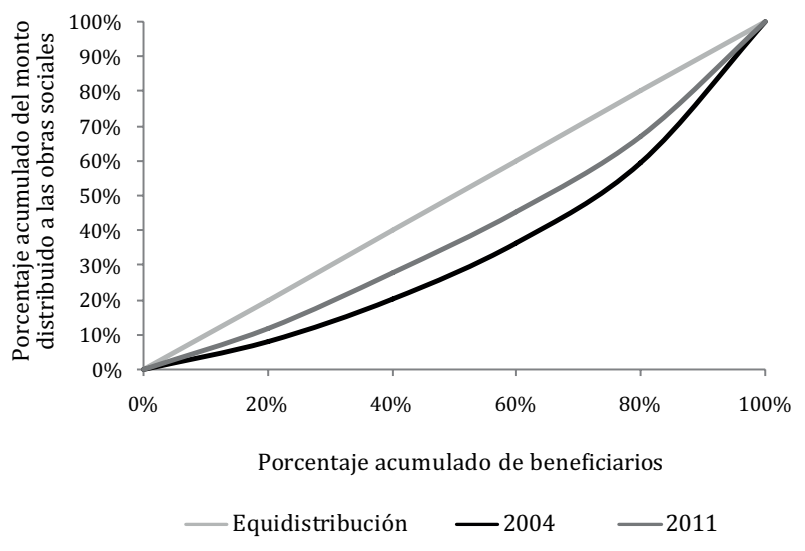

Figura 1. Curvas de Lorenz de la distribución de fondos entre beneficiarios de las obras sociales nacionales. Argentina, 2004-2011. 
población beneficiaria de obras sociales nacionales que traslada su aporte a una empresa de medicina prepaga.

Contrariamente a lo esperado, entre 2004 y 2011 no se reduce el número de entidades del subsistema de obras sociales nacionales. Las condiciones económicas favorables (con su impacto positivo sobre las finanzas de todas las entidades), así como la renuencia gubernamental a cerrar obras sociales con menor cantidad de afiliados y con posibles mayores dificultades financieras, son algunos factores que pueden explicar este resultado.

El elevado número de entidades contrasta con el carácter eminentemente concentrado del sistema: 20 entidades (entre 290 aproximadamente) obtienen valores cercanos al $60 \%$ de los cotizantes, beneficiarios y fondos en los dos años analizados. Dicho esto, se observa un aumento moderado en la concentración de cotizantes en las entidades más importantes (por el número de cotizantes), una caída en la concentración de los beneficiarios y una tendencia invariante en el caso de los fondos distribuidos.

El aumento de la concentración de cotizantes (y no de beneficiarios) podría atribuirse a la dinámica de los traspasos de afiliados y/o a las nuevas incorporaciones de trabajadores que ingresaron al mercado laboral formal.

Los traspasos entre obras sociales se producen especialmente por parte de jóvenes, solteros y sin hijos, que reciben las mejores ofertas para adquirir planes de cobertura diferenciales. Dado que en estos traspasos la relación entre beneficiarios y cotizantes es menor al promedio del sistema, las obras sociales receptoras podrían exhibir una reducción de su relación beneficiarios/cotizantes. Asumiendo que las principales obras sociales receptoras se encuentren en el ranking de las más importantes del sistema, los traspasos podrían acentuar la concentración de cotizantes y reducir la de beneficiarios.

Por otra parte, si en las nuevas incorporaciones al sistema de obras sociales también tienen un alto peso los jóvenes sin hijos o los segundos perceptores de ingresos del hogar, en la medida en que sean las entidades más importantes las beneficiadas por tales altas, el resultado también será una aumento de la concentración de afiliados y una caída de la de beneficiarios.

Por último, la falta de cambios en la concentración de fondos en las entidades más importantes (por número de cotizantes) es consistente con el accionar distributivo del Fondo Solidario de Redistribución.

Al tomar como unidad de análisis las obras sociales nacionales, agrupándolas en quintiles según el salario medio por cotizante de cada entidad, puede observarse una reducción de las diferencias entre los salarios promedios del primer y último quintil en el periodo estudiado. La dinámica del mercado formal de trabajo, con una disminución de las diferencias de los salarios de los distintos sectores productivos (o gremios), es el principal factor que podría explicar esta evolución. Sin embargo, también podría atribuirse al traspaso de aportantes de altos salarios a entidades de menores ingresos promedios. Si bien con los datos disponibles y la metodología de análisis empleada no es posible descartar en forma categórica esta última opción, resulta poco plausible en vista de los incentivos planteados por la desregulación: para que los traspasos entre entidades hayan constituido un factor de reducción de las desigualdades salariales, estos deberían haber beneficiado a las obras sociales más "pobres" en términos relativos, lo cual si bien es posible, resulta contraintuitivo.

Si bien distintos estudios sugieren que en sus primeros años de vigencia la libertad de elección de obras sociales promovió un proceso de descreme que podría haber acentuado las desigualdades en el financiamiento de las obras sociales nacionales, los resultados expuestos sugieren que en el periodo analizado la tendencia fue justamente la opuesta: las diferencias en los fondos recibidos en términos per cápita (considerando tanto cotizantes como beneficiarios) por las distintas entidades del sistema se redujo. Al menos dos factores complementarios pueden explicar este resultado.

En primer lugar, el rol desempeñado por el Fondo Solidario de Redistribución y, en particular, la incorporación de transferencias 
automáticas a las entidades considerando los ajustes por riesgo en función de la edad y sexo de los beneficiarios, pueden haber reducido los incentivos al descreme. Asimismo, la comparación en cada año estudiado de las diferencias salariales vs las diferencias en los fondos por cotizante o beneficiario ponen en evidencia su efectividad como herramienta de redistribución.

En segundo lugar, la tendencia hacia la reducción de la desigualdad en los salarios relativos del mercado de trabajo formal, como resultado del nuevo marco regulatorio (convenciones colectivas de trabajo) y de un contexto económico caracterizado por altas tasas de crecimiento y desempleo en baja, representó un factor decisivo sobre la conformación y dinámica de las obras sociales nacionales durante el periodo estudiado.

Por último, una debilidad del estudio realizado (y que debe ser tenida en cuenta al momento de valorar los resultados expuestos) es que el análisis de las desigualdades entre las obras sociales de los fondos disponibles por beneficiario y cotizantes no contemplan los pagos adicionales que hacen los usuarios al demandar planes diferenciales con mayores coberturas. Esta información no es provista por el ente recaudador, y podría esconder desigualdades al interior del sistema.

\section{NOTAS FINALES}

a. La entidades excluidas del estudio por no registrar continuidad en sus operaciones en los años analizados fueron para el año 2004: Personal de Maestranza; Personal de Azúcar Ingenio Las Toscas; Viajantes de Comercio; Árbitros del Fútbol Argentino; Asociación de Obras Sociales de Rosario; Empleados y Obreros Gastronómicos de Tucumán; Personal Jerárquico Industria Gráfica Argentina; Personal Artes Gráficas Chaco; Personal Artes Gráficas Santa Fe; Portuarios de Bahía Blanca; Portuarios Puerto San Nicolás. Para el año 2011: Personal Azúcar Ingenio Río Grande y Personal Auxiliar Casas Particulares.

b. Se considera asalariado a las personas que perciben un salario por la labor desempeñada en relación de dependencia. Asimismo, se considera registrado o con relación laboral formal a aquellos asalariados que tienen descuento jubilatorio por el trabajo desempeñado.

c. La paridez media final es una medida que da cuenta de la fecundidad. Se define como la relación entre los hijos nacidos vivos de mujeres de 45 a 49 años de edad a lo largo de su vida reproductiva (fecundidad retrospectiva) en relación al total de mujeres censadas en esas edades. Se trata de una medida propia del análisis longitudinal de la fecundidad.

\section{REFERENCIAS BIBLIOGRÁFICAS}

1. Ministerio de Salud. Políticas de salud [Internet]. Buenos Aires: MS; 2000 [citado 10 jul 2015]. Disponible en: http://goo.gl/WCmwCF.
2. Organización Panamericana de la Salud. Perfil de los sistemas y servicios de salud de Argentina [Internet]. Buenos Aires: OPS; 1998 [citado 10 jul 2015]. Disponible en: http://goo.gl/o4bWWs.

3. Programa de las Naciones Unidas para el Desarrollo. El sistema de salud argentino y su trayectoria de largo plazo: logros alcanzados y desafíos futuros [Internet]. Buenos Aires: PNUD; 2011 [citado 10 jul 2015]. Disponible en: http://goo.gl/gvlGvO.

4. Tobar F. Economía de la reforma de los seguros de salud en Argentina [Internet]. 2001 [citado 30 jun 2015]. Disponible en: http://goo.gl/m0MiOJ.

5. Médici A. La desregulación de las obras sociales: Un episodio más de la reforma de salud en Argentina... ¿qué vendrá luego? [Internet] Banco Interamericano de Desarrollo; 2002 [citado 15 jun 2015]. Disponible en: https:// goo.gl/PCORTw.

6. Findling L, Arruñada L, Klimovsky E. Desregulación y equidad: el proceso de reconversión de obras sociales en Argentina. Cadernos de Saúde Pública. 2002;18(4):1077-1086.

7. Tobar F, Mangiaterra A, Ventura G, Térmansen I. Endeudamiento sectorial [Internet]. Buenos Aires: Programa de Investigación Aplicada de Salud, Instituto Universitario Isalud; 2000 [citado 30 jun 2015]. Disponible en: http://goo.gl/eQ3ewt.

8. Jack W. Health insurance reform in four Latin American countries: theory and practice [Internet]. World Bank; 2000 [citado 25 jun 2010]. Disponible en: http:// goo.gl/9UKMjX. 
9. Fidalgo M. Adiós al derecho a la salud: El desarrollo de la medicina prepaga. Buenos Aires: Espacio; 2008.

10. Cetrángolo O, Devoto F. Organización de la salud en Argentina y equidad: Una reflexión sobre las reformas de los años noventa e impacto de la crisis actual [Internet]. 2002 [citado 10 jul 2015]. Disponible en: http://goo.gl/ X50kT2.

11. Groisman F. Empleo, salarios y desigualdad en Argentina: análisis de los determinantes distributivos. Problemas del Desarrollo. 2014;45(177):59-86.

12. World Health Organization. Global Health Observatory country views. Argentina statistics summary (2002 - present) [Internet]. c2015 [citado 5 jul 2015]. Disponible en: http://goo.gl/nR7YYI.

13. Instituto Nacional de Estadística y Censos. Censo Nacional de Población, Hogares y Viviendas 2001 [Internet]. Buenos Aires: INDEC [citado 30 jun 2015]. Disponible en: http://goo.gl/sLHG7l.

14. Instituto Nacional de Estadística y Censos. Censo Nacional de Población, Hogares y Viviendas 2010 [Internet]. Buenos Aires: INDEC [citado 30 jun 2015]. Disponible en: http://goo.gl/7UOK10.
15. Cavagnero E, Carrin G, Torres R. A National Health Insurance Plan for Argentina: simulating its financial feasibility [Internet]. World Health Organization; 2010 [citado 20 jun 2014]. Disponible en: http://goo.gl/ cp7l8u.

16. Administración Federal de Ingresos Públicos. Boletines Mensuales de Seguridad Social [Internet]. Buenos Aires: AFIP [citado 30 jun 2015]. Disponible en: https:// goo.gl/3qXtgo.

17. Ministerio de Economía, Dirección Nacional de Relaciones Económicas con las Provincias. Empleo privado formal en Argentina [Internet]. Buenos Aires: Ministerio de Economía; 2012 [citado 5 jul 2015]. Disponible en: http://goo.gl/OLKnOs.

18. Ministerio de Trabajo, Empleo y Seguridad Social; Subsecretaría de Programación Técnica y Estudios Laborales. Mejora en el nivel de empleo registrado del sector privado: Encuesta de Indicadores Laborales [Internet]. Buenos Aires: MTEySS; 2009 [citado 31 jul 2015]. Disponible en: http://goo.gl/fwMNbC.

FORMA DE CITAR

Arnaudo MF, Lago F, Moscoso N, Báscolo E, Yavich N. Concentración y desigualdades en el financiamiento de las obras sociales posdesregulación: un análisis comparativo de los años 2004 y 2011. Salud Colectiva. 2016;12(1):125-137

Recibido: 12 de agosto de 2015 | Versión final: 17 de noviembre de 2015 | Aprobado: 30 de noviembre de 2015

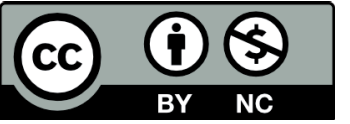

Este obra está bajo una licencia de Creative Commons Reconocimiento-NoComercial 4.0 Internacional. Reconocimiento - Permite copiar, distribuir y comunicar públicamente la obra. A cambio, se debe reconocer y citar al autor original. No Comercial - Esta obra no puede ser utilizada con finalidades comerciales, a menos que se obtenga el permiso.

http://dx.doi.org/10.18294/sc.2016.880 